\title{
Long-term outcomes of liver transplantation in patients with hepatitis $C$ infection are not affected by HCV positivity of a donor
}

Maria Stepanova ${ }^{1,2}$, Mehmet Sayiner ${ }^{1}$, Leyla de Avila ${ }^{1}$, Zahra Younoszai ${ }^{1}$, Andrei Racila ${ }^{1}$ and Zobair M. Younossi ${ }^{1,3^{*}}$

\begin{abstract}
Background: The use of HCV-positive livers for HCV-positive recipients is becoming more common. Our aim is to evaluate long-term outcomes in liver transplant recipients transplanted with HCV antibody-positive organs.

Methods: From the Scientific Registry of Transplant Recipients (1995-2013), we selected all adult liver transplant recipients with $\mathrm{HCV}$, and cross-sectionally compared long-term graft loss and mortality rates between those who were transplanted from HCV antibody-positive $(\mathrm{HCV}+)$ vs. HCV antibody-negative donors.

Results: We included 33,668 HCV+ liver transplant recipients (54.0 \pm 7.7 years old, $74.1 \%$ male, $71.0 \%$ white, $23.6 \%$ with liver malignancy). Of those, $5.7 \%(\mathrm{~N}=1930)$ were transplanted from HCV+ donors; the proportion gradually increased from 2.9\% in 1995 to $9.4 \%$ in 2013. Patients who were transplanted from HCV+ positive donors were more likely to be discharged alive after transplantation ( $95.4 \%$ vs. $93.9 \%, p=0.006)$, but this difference was completely accounted for by a greater proportion of HCV+ donors in more recent study years ( $p=0.10$ after adjustment for the transplant year). After transplantation, both mortality in HCV patients transplanted from HCV+ donors (12.5\% in 1 year, $24.2 \%$ in 3 years, $33.0 \%$ in 5 years) and the graft loss rate (2.2\% in 1 year, $4.8 \%$ in 3 years, $7.5 \%$ in 5 years) were similar to those in HCV patients transplanted from HCV-negative donors (all $p>0.05$ ).

Conclusions: Over the past two decades, the use of HCV+ organs for liver transplantation has tripled. Despite this, the long-term outcomes of HCV+ liver transplant recipients transplanted from HCV+ donors were not different from those who were transplanted with HCV-negative organs.
\end{abstract}

Keywords: Liver transplant, HCV, Extended criteria donor

\section{Background}

Chronic hepatitis C infection is currently the most common indication for liver transplantation in the U.S. [1]. After the disease progresses to its most advanced stages which include hepatocellular carcinoma and liver failure, the only alternative to liver-related death is receiving a liver transplant. Since the progression rate increases with age [2], and given that the highest prevalence of $\mathrm{HCV}$

\footnotetext{
* Correspondence: zobair.younossi@inova.org

${ }^{1}$ Betty and Guy Beatty Center for Integrated Research, Inova Health System, Claude Moore Health Education and Research Building 3300 Gallows Road, Falls Church, VA 22042, USA

${ }^{3}$ Center for Liver Diseases, Department of Medicine, Inova Fairfax Hospital, Falls Church, VA, USA

Full list of author information is available at the end of the article
}

infection in the U.S. has been reported in the baby boomer population [3] who are currently between 50 and 70 years of age, the need for liver transplantation in $\mathrm{HCV}$-infected patients is likely to remain high in the coming years. It is unclear whether the recently approved direct-acting antiviral (DAA) regimens for $\mathrm{HCV}$, for which their high efficacy and minimal contraindications are at this point counter-balanced by accessrelated barriers [4-6], are able to reverse this trend at the national level any time soon.

Due to the shortage of organs, the use of HCVinfected grafts has long been considered as a potentially viable alternative for patients who are already infected with HCV. Although concerns about the presence of 
HCV-related damage to the graft and about viral transmission initially seemed plausible, in the last two decades, an increasing number of centers have adopted the practice of extending their criteria to include HCVpositive donors for using in $\mathrm{HCV}$-positive recipients. As of now, the results reported from both single-center and multi-center studies have been largely consistent about the lack of an additional risk to a recipient associated with the presence of $\mathrm{HCV}$ infection in a graft, given that both a recipient and a donor meet other selection criteria [7-11]. It is important to note that most of these studies had a relatively short follow-up. On the other hand, the results reported from other multi-center studies have suggested that the use of HCV-positive donors is not truly risk-free and may, in fact, result in an additional clinical burden which includes an increased risk of developing advanced post-transplant fibrosis and more rapid recurrence of hepatitis in the graft $[12,13]$. It is unclear whether this burden could eventually translate into compromised long-term outcomes with long enough follow-up.

The aim of this study is to report post-transplant outcomes in HCV-positive recipients transplanted from $\mathrm{HCV}$-positive donors, and to evaluate the risk for mortality and graft loss associated with the use of HCVpositive donors, using the most recent long-term follow-up data from a nationwide registry of liver transplant recipients.

\section{Methods}

\section{Study cohort}

This study used data from the Scientific Registry of Transplant Recipients (SRTR). The SRTR data system includes data on all donor, wait-listed candidates, and transplant recipients in the U.S., submitted by the members of the Organ Procurement and Transplantation Network (OPTN), and has been described elsewhere. The Health Resources and Services Administration (HRSA), U.S. Department of Health and Human Services provides oversight to the activities of the OPTN and SRTR contractors.

In this study, we included all liver transplant recipients with $\mathrm{HCV}$ of at least 18 years of age and older who underwent liver transplantation between 1995 and 2013 in the U.S. In both recipients and donors, their anti$\mathrm{HCV}$ serology status was used to define those with and without $\mathrm{HCV}$ ( $\mathrm{HCV}+$ and $\mathrm{HCV}-$, respectively). No $\mathrm{HCV}$ RNA testing was reported in SRTR.

The length of hospital stay for patients following liver transplantation was calculated in days from the date of transplant to the date of discharge. Inpatient mortality and history of acute rejection during inpatient stay were recorded. We also collected information on organ donors that included high risk donors classified by the
CDC criteria, basic demographics and clinical history (diabetes, cancer), and recorded procurement from a non-heart beating donor.

The primary outcomes included in this study were inpatient mortality, post-transplant mortality (calculated by matching with the Social Security Death Master File, provided by SRTR), and post-transplant graft failure (defined as a documented re-transplant or a graft loss-related cause of death). Patients undergoing retransplantation were included in the mortality analysis with their most recent transplants only.

Liver transplant recipients discharged alive were followed at 6 and 12 months post-transplant, and then annually until death, re-transplantation, or loss to follow-up. Patients with no documented date of death were presumed alive as of September 1, 2015. The time to events for survival analysis was calculated from the date of transplant.

\section{Statistical analysis}

The clinico-demographic parameters and outcomes of liver transplant recipients with $\mathrm{HCV}+$ were compared between those transplanted from $\mathrm{HCV}+$ and $\mathrm{HCV}$ - donors using a Kruskall-Wallis non-parametric test and a chisquare test for homogeneity. The association of a donor's $\mathrm{HCV}$ positivity with time to post-transplant mortality and graft failure was evaluated by a Cox proportional hazard model with adjustment for clinical and demographic parameters of both donors' and recipients' and for immunosuppressant medications used in follow-up. $P$-values of 0.05 or lower were considered statistically significant. All analyses were run in SAS 9.3 (SAS Institute, Cary, NC).

For this study, a data use agreement with SRTR was signed, and a permission to publish the manuscript was obtained. The study was granted a non-human subject research exemption by Inova Institutional Review Board.

\section{Results}

There were 106,427 liver transplants conducted between 1995 and 2013 included in SRTR. Of those, 95,710 were in individuals of at least 18 years of age, and in 75,469 of those the HCV status of a recipient, the HCV status of a donor, and a recipient's mortality status were available. Of this subpopulation, 33,668 (44.6\%) liver transplant recipients were $\mathrm{HCV}+$.

There was a substantial increase in the proportion of $\mathrm{HCV}+$ recipients being transplanted from $\mathrm{HCV}+$ donors during the study years. Indeed, the increase in less than 20 years was more than three-fold, from less than $3 \%$ in 1995 to more than $9 \%$ in 2013. The greatest change in the trend happened in 2010 which resulted in a nearly $50 \%$ increase in the proportion of $\mathrm{HCV}+$ donors used for transplantation in the following 3 years, by at least $10 \%$ annually (Fig. 1). 


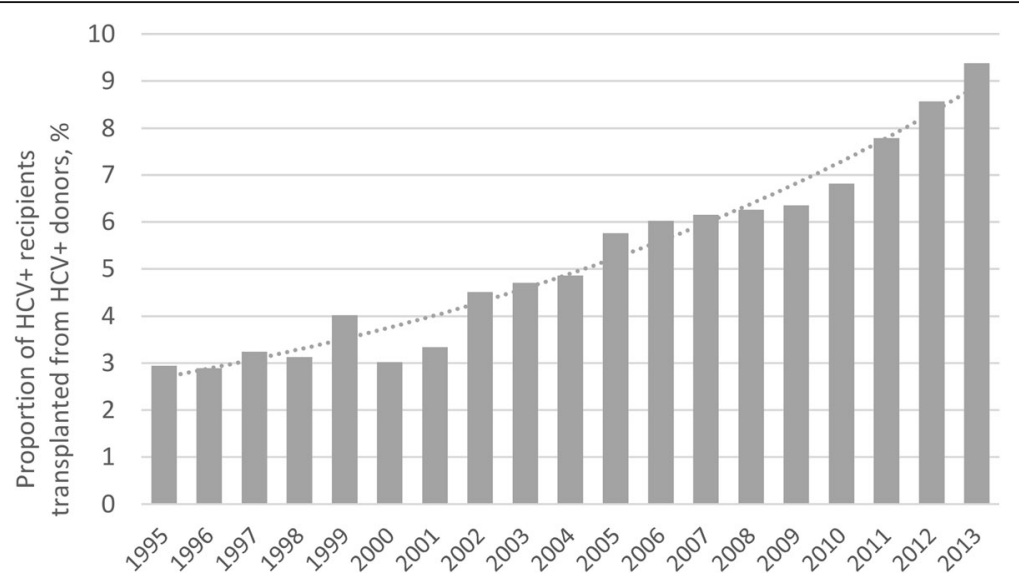

Fig. 1 The proportion of HCV+ donors used for transplantation in HCV+ recipients by year

Comparison of clinico-demographic parameters of $\mathrm{HCV}+$ liver transplant recipients transplanted from $\mathrm{HCV}+$ and HCV- donors is included in Table 1. There was a greater proportion of African-American patients receiving $\mathrm{HCV}+$ transplants, as well as more patients with pre-transplant type 2 diabetes, liver cancer, and a greater proportion of re-transplants (all $p<0.05$ ) (Table 1). Despite this, patients transplanted with HCV+ organs had lower MELD scores both with and without adjustment for the presence of liver cancer (both $p<0.0001$ ).

The HCV+ donors were, on average, older, less frequently non-heart-beating at the time of procurement, and were substantially more frequently classified as high risk (all $p<0.0001$ ). There was no difference in donors' gender and history of diabetes or cancer (all $p>0.05$ ) (Table 1).

The short-term outcomes of $\mathrm{HCV}+$ liver transplant recipients were more favorable in those transplanted from $\mathrm{HCV}+$ donors, including a lower rate of inpatient acute rejection events and inpatient mortality, and a shorter inpatient stay (all $p<0.007)$ (Table 1$)$. However, when adjusted for the year of transplant, no difference in any of those outcomes was found (all $p>0.10$ ).

Long-term outcomes were similar in patients transplanted from $\mathrm{HCV}+$ and HCV- donors (Table 1, Fig. 2). Indeed, a borderline significant difference was observed for 1 and 2 years post-transplant mortality ( 1 year: $12.5 \%$ in patients transplanted from $\mathrm{HCV}+$ donors vs. $14.1 \%$ in patients with $\mathrm{HCV}$ - donors, $p=0.059 ; 2$ years: $18.4 \%$ vs. $20.2 \%, p=0.054$ ) which was again completely explained out by the bias in the year of transplant (both $p>0.21$ after adjustment for year). At the same time, the following years after transplantation revealed nearly identical mortality rates (all $p>0.5$ ).

Similarly, the graft loss rates were insignificantly lower in patients transplanted from $\mathrm{HCV}+$ donors up to 3 years post-transplant $(2.2 \%$ vs. $2.8 \%$ at 1 year, $p=0.15 ; 3.6 \%$ vs. $4.5 \%$ at 2 years, $p=0.090 ; 4.8 \%$ vs. $5.9 \%$ at 3 years, $p=$ 0.089 ) due the yearly distribution of $\mathrm{HCV}+$ donors (all $p>0.13$ after adjustment for year).

In a Cox proportional hazard model, after adjustment for the year of transplant, recipient's age, gender, race, elements of medical history, MELD score, donor's age and medical history, and the use of immunosuppressant medications, the association of a donor's $\mathrm{HCV}+$ status with post-transplant graft loss and mortality in $\mathrm{HCV}+$ liver transplant recipients was highly insignificant: adjusted hazard ratio (aHR) $(95 \% \mathrm{CI})=1.045$ (0.954-1.145) for time to post-transplant mortality, aHR $=0.922(0.727-1.169)$ for time to graft loss (both $p>0.33$ ) (all mortality and graft loss predictors tested in the Cox proportional hazard models are included in Additional file 1: Table Table S1). Furthermore, the lack of association of donors' HCV positivity with the studied outcomes was also confirmed in a separate round of case-control analysis where the outcomes of patients transplanted from $\mathrm{HCV}+$ donors were compared to those of propensity score-matched controls (all $p>0.15$ ) (Additional file 2: Table S2). In contrast, a more recent year of transplant was a major predictor of both lower mortality rate $(\mathrm{aHR}=0.963$ (0.956-0.971) per year) and lower graft failure rate $($ aHR $=0.950(0.934-0.967)$ per year $)($ both $p<0.0001)$ even after adjustment for the type of immunosuppressants used in follow-up (Additional file 2: Table S2).

\section{Discussion}

In this study, we reported post-transplant outcomes in patients with $\mathrm{HCV}$ transplanted from $\mathrm{HCV}$ antibodypositive donors and compared these outcomes to those of $\mathrm{HCV}$ patients transplanted from $\mathrm{HCV}$-negative donors using 20 years of data from the nationwide transplant registry. Our study shows that there are no differences in 
Table 1 Comparison of HCV+ liver transplant recipients, donors and outcomes in transplantations from HCV+ and HCV-donors

\begin{tabular}{|c|c|c|c|}
\hline & $\mathrm{HCV}+$ donor & HCV- donor & $P$ \\
\hline N & 1930 & 31,738 & \\
\hline \multicolumn{4}{|l|}{ Recipients: } \\
\hline Age, years & $55.1 \pm 7.2$ & $53.9 \pm 7.7$ & $<0.0001$ \\
\hline Male gender & $1448(75.0 \%)$ & $23,508(74.1 \%)$ & 0.35 \\
\hline Race/ethnicity: Caucasian & $1346(69.7 \%)$ & $22,575(71.1 \%)$ & 0.19 \\
\hline Race/ethnicity: African-American & $267(13.8 \%)$ & 3249 (10.2\%) & $<0.0001$ \\
\hline Race/ethnicity: Hispanic & $254(13.2 \%)$ & $4664(14.7 \%)$ & 0.06 \\
\hline Co-infected with HBV (HBV sAg+) & $51(2.7 \%)$ & 959 (3.1\%) & 0.32 \\
\hline Pre-transplant history of type 2 diabetes & $236(13.3 \%)$ & 3335 (11.7\%) & 0.0441 \\
\hline Liver cancer & $544(28.2 \%)$ & $7414(23.4 \%)$ & $<0.0001$ \\
\hline Liver re-transplant & $3(0.2 \%)$ & $6(0.0 \%)$ & 0.0004 \\
\hline MELD score & $18.2 \pm 8.3$ & $20.3 \pm 9.7$ & $<0.0001$ \\
\hline MELD score excl. liver cancer & $19.9 \pm 8.3$ & $22.4 \pm 9.5$ & $<0.0001$ \\
\hline \multicolumn{4}{|l|}{ Donors: } \\
\hline Age, years & $42.0 \pm 11.8$ & $39.6 \pm 16.4$ & $<0.0001$ \\
\hline Male gender & $1196(62.0 \%)$ & $19,513(61.5 \%)$ & 0.67 \\
\hline Non-heart-beating & $31(1.6 \%)$ & $1138(3.6 \%)$ & $<0.0001$ \\
\hline History of diabetes & $167(8.7 \%)$ & $2491(7.9 \%)$ & 0.19 \\
\hline History of cancer & $39(2.0 \%)$ & $834(2.6 \%)$ & 0.11 \\
\hline History of high risk behavior & $808(42.4 \%)$ & $2044(6.5 \%)$ & $<0.0001$ \\
\hline Heterotopic transplant & $2(0.1 \%)$ & $35(0.1 \%)$ & 0.95 \\
\hline \multicolumn{4}{|l|}{ Outcomes: } \\
\hline Acute rejection episodes before discharge & $36(2.4 \%)$ & $809(4.1 \%)$ & 0.0020 \\
\hline Discharged alive & $1842(95.4 \%)$ & $29,808(93.9 \%)$ & 0.0063 \\
\hline Length of inpatient stay, days & $15.7 \pm 20.3$ & $16.4 \pm 21.9$ & 0.0013 \\
\hline Mortality: 1 year & $242(12.5 \%)$ & 4467 (14.1\%) & 0.059 \\
\hline Mortality: 3 years & $399(24.2 \%)$ & $7190(24.8 \%)$ & 0.58 \\
\hline Mortality: 5 years & $424(33.0 \%)$ & $8028(32.5 \%)$ & 0.68 \\
\hline Mortality: 10 years & $281(47.5 \%)$ & $7019(48.6 \%)$ & 0.59 \\
\hline Graft failure: 1 year & $38(2.2 \%)$ & $782(2.8 \%)$ & 0.15 \\
\hline Graft failure: 3 years & $63(4.8 \%)$ & 1375 (5.9\%) & 0.09 \\
\hline Graft failure: 5 years & $70(7.5 \%)$ & $1465(8.1 \%)$ & 0.56 \\
\hline Graft failure: 10 years & $50(13.9 \%)$ & 1160 (13.5\%) & 0.86 \\
\hline
\end{tabular}

both short- and long-term outcomes between patients who were transplanted from $\mathrm{HCV}$-positive and $\mathrm{HCV}$ negative donors. This is largely consistent with prior reports of similar findings reported from smaller studies with shorter follow-up.

This finding provides an additional support to the practice of using $\mathrm{HCV}$-positive donors for $\mathrm{HCV}$-positive recipients which has become nearly universal in multiple transplant centers. Indeed, we have shown that the use of HCV-positive organs has tripled over less than two decades. Nevertheless, our data confirm that the medium-term outcomes of patients with $\mathrm{HCV}$ are similar regardless of $\mathrm{HCV}$ positivity of the organs. Furthermore, the short-term outcomes, including being discharged alive from the hospital, having a rejection episode before discharge, and 1 year mortality, all seem to be lower in HCV-positive organ recipients. This superior short-term survival, in addition to the impact of a greater proportion of patients receiving $\mathrm{HCV}$-positive organs in the most recent study years, may also be related to less advanced liver disease in these patients suggesting a cautious use of $\mathrm{HCV}$-positive organs. Indeed, patients receiving $\mathrm{HCV}$-positive organs had lower MELD scores which remained true even after excluding those 


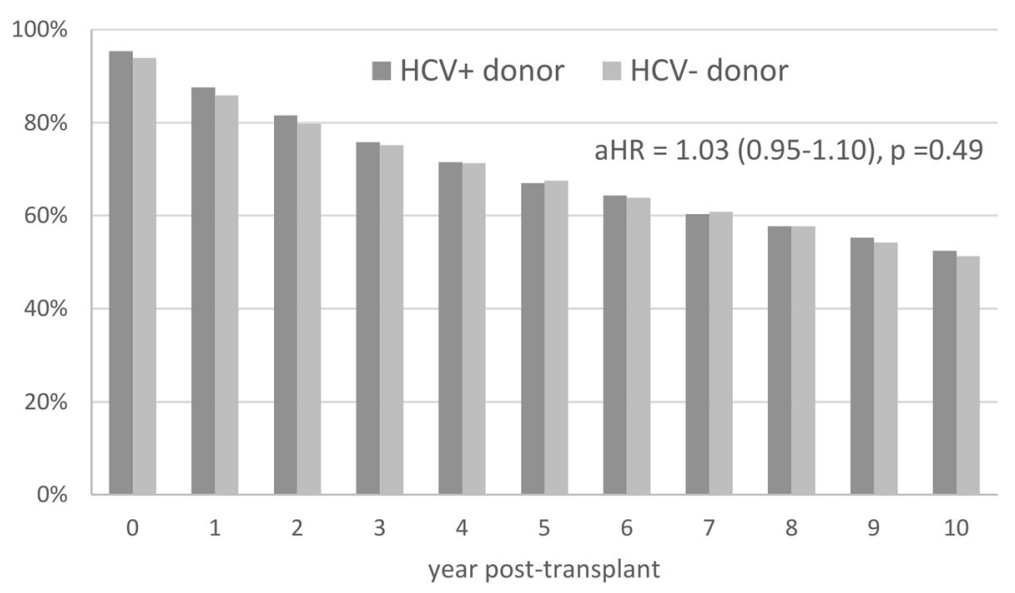

Fig. 2 Post-transplant survival in HCV+ liver transplant recipients transplanted from HCV+ and HCV- donors. P> 0.05 at all time points. aHR adjusted hazard ratio. Mortality at the zero time point represents inpatient mortality at transplant

who received MELD exclusion for hepatocellular carcinoma (HCC).

While there seems to be no evidence supporting the presence of an increased risk of post-transplant mortality and allograft losses arisen solely from the HCV-positivity of a donor, this finding should be interpreted with caution. In particular, it is necessary to understand that hepatocytes in an $\mathrm{HCV}$-positive graft might still have been injured by chronic inflammation, so a careful selection of both grafts and recipients will be important [13]. Nevertheless, it is interesting to note that in our study, we have failed to find any evidence of an increased risk of adverse post-transplant outcomes associated with being HCV-positive in the group of donors age 45 or older or in the donors with history of type 2 diabetes (all $p>0.05$ ).

With the new DAAs becoming more widely available, the utility of $\mathrm{HCV}$-seropositive organs becomes even more important. Indeed, we suggest that the use of $\mathrm{HCV}$-positive organs in HCV-positive recipients who can be safely and effectively treated post-transplant will become increasingly a viable option. However, it is important to note that although preliminary studies have shown notable benefits of treating post-transplant $\mathrm{HCV}$ patients with DAAs $[14,15]$, long-term data is still not available.

The study did have a limitation related to the lack of HCV RNA results for the donors, so the impact of active viremia in both donors and recipients could not be evaluated, while it is reasonable to believe that such impact may exist. We also did not have access to histology data for the grafts so the risk associated with the presence and/or degree of hepatic steatosis and fibrosis in an allograft could not be studied. The outcomes were limited to mortality and graft loss only, while other clinical outcomes, such as developing hepatic inflammation and fibrosis, could also significantly affect patients' wellbeing in the post-transplant period.

\section{Conclusions}

In this study which used the most recent nationwide registry of liver transplant recipients, we have found no evidence of an increased risk for adverse post-transplant outcomes that could be associated with $\mathrm{HCV}$ positivity of a donor. Therefore, we postulate that while we, as a society, are working on decreasing wait-list mortality in liver transplant candidates, HCV-positive allografts could be a reasonably safe option for patients with chronic HCV infection who are in need of a liver transplant. It is important to note that our data cannot be used in support of indiscriminate use of $\mathrm{HCV}$-positive donors, especially those who are HCV RNA-positive, given previously reported unfavorable histologic outcomes which could not be ruled out in this study. Further studies are needed to establish evidence-based selection criteria for $\mathrm{HCV}$ positive donors which could provide patients with the best possible risk-to-benefit ratio.

\section{Additional files}

Additional file 1: Table S1. Independent predictors of post-discharge mortality and graft loss in patients with HCV. (DOCX $18 \mathrm{~kb}$ )

Additional file 2: Table S2. Case-control comparison of propensity score-matched HCV patients transplanted from HCV+ and HCV- donors. (DOCX $18 \mathrm{~kb})$

\section{Abbreviations}

aHR: Adjusted hazard ratio; BMI: Body mass index; COPD: Chronic obstructive pulmonary disease; CVD: Cardiovascular disease; DAA: Direct-acting antiviral; HBV: Hepatitis B virus; HCC: Hepatocellular carcinoma; HCV: Hepatitis C virus; ICU: Intensive care unit; MELD: The model for end-stage liver disease; SRTR: The Scientific Registry of Transplant Recipients 


\section{Acknowledgements}

The data reported here have been supplied by the Minneapolis Medical Research Foundation (MMRF) as the contractor for the Scientific Registry of Transplant Recipients (SRTR). The interpretation and reporting of these data are the responsibility of the authors and in no way should be seen as an official policy of or interpretation by the SRTR or the U.S. Government.

\section{Funding}

This study was supported by the Beatty Liver and Obesity Research Fund and Liver Outcomes Research Fund, Inova Health System, Falls Church, VA.

\section{Availability of data and materials}

The data used in this study can be requested from SRTR for a fee. Per DUA between the authors and SRTR, deposition of the data in publicly available repositories is not allowed.

\section{Authors' contributions}

MaS - study concept and design, analysis and interpretation of data; drafting of the manuscript. MeS, LdA, ZaY, AR - critical revision of the manuscript for important intellectual content. ZoY - study concept and design; critical revision of the manuscript for important intellectual content; obtained funding; study supervision. All authors read and approved the final manuscript.

\section{Competing interests}

Maria Stepanova is an active Associate Editor for BMC Gastroenterology. Other coauthors have no conflict of interest related to this manuscript to disclose.

\section{Consent for publication}

Not applicable.

\section{Ethics approval and consent to participate}

Per Data Use Agreement (DUA) with SRTR, the authors were required to use the data solely for bona fide research/analysis described in a pre-approved research plan, and were specifically required not use the data for any commercial purpose which could have a negative impact on patient welfare, such as offering, denying, or allocating insurance; and adverse selection (e.g., identifying patients with high risk diagnoses). The text of the manuscript was reviewed by SRTR to ensure compliance with the terms of the DUA regarding confidentiality, and approved for publication. The study was granted a non-human subject research status by Inova Institutional Review Board.

\section{Author details}

'Betty and Guy Beatty Center for Integrated Research, Inova Health System, Claude Moore Health Education and Research Building 3300 Gallows Road, Falls Church, VA 22042, USA. ${ }^{2}$ Center for Outcomes Research in Liver Diseases, Washington, DC, USA. ${ }^{3}$ Center for Liver Diseases, Department of Medicine, Inova Fairfax Hospital, Falls Church, VA, USA.

Received: 20 May 2016 Accepted: 29 October 2016 Published online: 15 November 2016

\section{References}

1. Stepanova M, Wai H, Saab S, Mishra A, Venkatesan C, Younossi ZM. The portrait of an adult liver transplant recipient in the United States from 1987 to 2013. JAMA Intern Med. 2014:174(8):1407-9.

2. Westbrook RH, Dusheiko G. Natural history of hepatitis C. J Hepatol. 2014; 61(1 Suppl):S58-68.

3. Coilly A, Roche B, Duclos-Vallée JC, Samuel D. News and challenges in the treatment of hepatitis C in liver transplantation. Liver Int. 2016;36 Suppl 1: 34-42. doi:10.1111/liv.13017. Review.

4. Stepanova M, Younossi ZM. Interferon-free regimens for chronic hepatitis C: barriers due to treatment candidacy and insurance coverage. Dig Dis Sci. 2015:60(11):3248-51

5. Adinolfi LE, Guerrera B. All-oral interferon-free treatments: the end of hepatitis $C$ virus story, the dream and the reality. World J Hepatol. 2015; 7(22):2363-8.

6. Do A, Mittal Y, Liapakis A, Cohen E, Chau H, Bertuccio C, Sapir D, Wright J, Eggers C, Drozd K, Ciarleglio M, Deng Y, Lim JK. Drug authorization for sofosbuvir/ledipasvir (Harvoni) for chronic HCV infection in a real-world cohort: a new barrier in the HCV care cascade. PLoS One. 2015;10(8), e0135645.

7. Plotkin JS, Ridge L, Kuo PC, Lim J, Njoku MJ, Johnson LB. Extending the boundaries of acceptable organ donors: a means of expanding the donor pool for liver transplantation. Transplant Proc. 1997;29(8):3288.

8. Marroquin CE, Marino G, Kuo PC, Plotkin JS, Rustgi VK, Lu AD, Edwards E, Taranto S, Johnson LB. Transplantation of hepatitis C-positive livers in hepatitis C-positive patients is equivalent to transplanting hepatitis Cnegative livers. Liver Transpl. 2001;7(9):762-8.

9. Burr AT, Li Y, Tseng JF, Saidi RF, Bozorgzadeh A, Shah SA. Survival after liver transplantation using hepatitis C virus-positive donor allografts: casecontrolled analysis of the UNOS database. World J Surg. 2011;35(7):1590-5.

10. Álvaro E, Abradelo M, Fuertes A, Manrique A, Colina F, Alegre C, Calvo J, García M, García-Sesma A, Cambra F, Sanabria R, Moreno E, Jimenez C. Liver transplantation from anti-hepatitis $C$ virus-positive donors: our experience. Transplant Proc. 2012;44(6):1475-8.

11. Northup PG, Argo CK, Nguyen DT, McBride MA, Kumer SC, Schmitt TM, Pruett TL. Liver allografts from hepatitis C positive donors can offer good outcomes in hepatitis C positive recipients: a US National Transplant Registry analysis. Transpl Int. 2010;23(10):1038-44.

12. Ballarin R, Cucchetti A, Spaggiari M, Montalti R, Di Benedetto F, Nadalin S, Troisi RI, Valmasoni M, Longo C, De Ruvo N, Cautero N, Cillo U, Pinna AD, Burra P, Gerunda GE. Long-term follow-up and outcome of liver transplantation from anti-hepatitis C virus-positive donors: a European multicentric case-control study. Transplantation. 2011;91(11):1265-72.

13. Lai JC, O'Leary JG, Trotter JF, Verna EC, Brown Jr RS, Stravitz RT, Duman JD, Forman LM, Terrault NA, Consortium to Study Health Outcomes in HCV Liver Transplant Recipients (CRUSH-C). Risk of advanced fibrosis with grafts from hepatitis $C$ antibody-positive donors: a multicenter cohort study. Liver Transpl. 2012;18(5):532-8.

14. Saab S, Greenberg A, Li E, Bau SN, Durazo F, El-Kabany M, Han S, Busuttil RW. Sofosbuvir and simeprevir is effective for recurrent hepatitis $C$ in liver transplant recipients. Liver Int. 2015:35(11):2442-7.

15. Brown RS Jr, O'Leary JG, Reddy KR, Kuo A, Morelli GJ, Burton JR Jr, Stravitz RT, Durand C, Di Bisceglie AM, Kwo P, Frenette CT, Stewart TG, Nelson DR, Fried MW, Terrault NA; HCV-TARGET Study Group. Interferon-free therapy for genotype 1 hepatitis $C$ in liver transplant recipients: real world experience from HCV-TARGET. Liver Transpl. 2015. doi: 10.1002/It.24366

\section{Submit your next manuscript to BioMed Central and we will help you at every step:}

- We accept pre-submission inquiries

- Our selector tool helps you to find the most relevant journal

- We provide round the clock customer support

- Convenient online submission

- Thorough peer review

- Inclusion in PubMed and all major indexing services

- Maximum visibility for your research

Submit your manuscript at www.biomedcentral.com/submit
Biomed Central 